\title{
Magnetic Fields of Neutron Stars
}

\author{
Sushan Konar* \\ NCRA-TIFR, Pune, 411007, India \\ ${ }^{*}$ Corresponding author. E-mail: sushan@ncra.tifr.res.in
}

\begin{abstract}
This article briefly reviews our current understanding of the evolution of magnetic fields in neutron stars, which basically defines the evolutionary pathways between different observational classes of neutron stars. The emphasis here is on the evolution in binary systems and the newly emergent classes of millisecond pulsars.
\end{abstract}

Key words. neutron stars : population, magnetic fields—X-ray binaries : evolution—millisecond pulsars : interconnections

\section{Introduction}

Over the decades, one of the primary preoccupations of neutron star research has been to look for a unification scheme connecting the widely different observational classes (shown in Fig.1). The magnetic field, ranging from $10^{8} \mathrm{G}$ in millisecond pulsars to $10^{15} \mathrm{G}$ in magnetars, has been central to this theme as it plays an important role in determining the evolution of the spin, the radiative properties and the interaction of a neutron star with its surrounding medium. Consequently, it is the evolution of the magnetic field which links different observational classes through unique evolutionary pathways (Bhattacharya \& Srinivasan 1995; Bhattacharya 2002; Reisenegger 2007).

The nature of field evolution depends crucially on the material properties of the region where currents supporting the field are located. In the solid crust, the currents are carried by electrons making Ohmic diffusion and Hall drift the predominant mechanisms for field evolution (Goldreich \& Reisenegger 1992). While Ohmic diffusion is intrinsically dissipative (Sang \& Chan mugam 1987; Urpin \& Muslimov 1992; Urpin \& van Riper 1993; Geppert \& Urpin 1994; Urpin \& Geppert 1995; Konar \& Bhattacharya 1997; Konar \& Bhattachary 1999a; Urpin \& Konenkov 2008), Hall drift is basically an advection of the magnetic field. Although Hall drift is not a dissipative process, it can cause rearrangements of the field which may then enhance Ohmic dissipation (Hollerbach \& Rüdiger 2002; Rheinhardt \& Geppert 2002; Geppert \& Rheinhardt 2002; Reisenegger et al. 2007; Pons, Miralles, \& Geppert 2009; Viganò 2013).

On the other hand, in the core of a neutron star the electrical conductivities are so large that Hall drift and Ohmic diffusion are extremely slow to have any effect on astrophysically relevant time-scales. However, the core is a fluid mixture of electrons, protons and neutrons (and perhaps other more exotic particles) and the magnetic flux can be transported and re-arranged by convective motions for a period immediately after the birth of the neutron star. At later times (when the temperature is low enough) the charged particles and the associated magnetic field can drift with respect to the neutrons, in a process called 'ambipolar diffusion' (Goldreich \& Reisenegger 1992; Passamonti et al. 2017).

However, protons inside the core of a neutron star are expected to behave like a type-II superconductor with a upper critical field of $\sim 10^{16} \mathrm{G}$. In this case, magnetic field would exist in the form of an array of Abrikosov fluxoids. Recently, the MHD behaviour of such superconductors has been investigated and it appears that the time-scales of field evolution are extremely long (Glampedakis, Andersson, \& Samuelsson 2011; Graber et al. 2015; Elfritz et al. 2016). Therefore, the processes responsible for field evolution can only be effective if the currents supporting the field are located (or are relocated in the course of evolution) in the crustal region which has metal-like transport properties. A number of mechanisms have been proposed for the expulsion of magnetic flux from the core to the crust (Muslimov \& Tsygan 1985b; Srinivasan et al. 1990; Konenkov \& Geppert 2000) without reaching any consensus.

The evolution of the magnetic field is also significantly affected by external processes like material accretion. For example, the accretion phase connects up ordinary radio pulsars with their millisecond counterparts through a well defined evolutionary pathway. The rotationpowered millisecond pulsars are understood to acquire their low magnetic fields and fast spin frequencies through mass accretion in LMXBs. For this pathway to work, it has been argued that a physical model of field evolution should satisfy the following observational con- 
straints - relatively little magnetic field decay should take place in an isolated radio pulsar, while accretion should be able to reduce the surface field strength by several orders of magnitude (Bhattacharya 2002; Konar 2013). On the other hand, to understand the connection between different types of isolated neutron stars a detailed theory of magneto-thermal evolution has been developed in recent years (Pons, Miralles, \& Geppert 2009; Kaspi 2010; Viganò 2013). The resulting synergy between the theoretical models and the observation has indeed strengthened the notion of a magnetic field dominated evolutionary link between different observational classes of neutron stars.

\section{The Neutron Star Menagerie}

About 3000 neutron stars, with diverse characteristic properties, have been observed till date, in different parts of the electromagnetic spectrum. Interestingly, these can be classified into the three following types, according to the nature of of energy generation processes in them (Kaspi 2010; Konar 2013; Konar et al. 2016).

Rotation Powered Pulsars (RPP) : Discovered serendipitously (Hewish et al. 1968) and originally known as radio pulsars, these are powered by the loss of rotational energy due to magnetic braking. The basic observed quantities of a pulsar are the spin-period $\left(\mathrm{P}_{\mathrm{s}}\right)$ and the period derivative $(\dot{P})$, and the most important derived quantity is the dipolar component of the surface magnetic field given by (Manchester \& Taylor 1977),

$B_{\mathrm{s}} \simeq 3.2 \times 10^{19}\left(\frac{P_{\mathrm{s}}}{s}\right)^{\frac{1}{2}}\left(\frac{\dot{P}}{s s^{-1}}\right)^{\frac{1}{2}} \mathrm{G}$.

The RPPs again have three sub-classes.

1. Primary among the RPPs are the classical radio pulsars $(\mathrm{PSR})$ with $\mathrm{P}_{\mathrm{s}} \sim 1 \mathrm{~s}$ and $\mathrm{B}_{\mathrm{s}} \sim 10^{11.5}$ $10^{13.5} \mathrm{G}$.

2. Detection of the $1.5 \mathrm{~ms}$ pulsar B1937+21 (Backer et al. 1982) heralded this new genre of millisecond radio pulsars (MSRP). MSRPs are usually defined as the ones with $P<20-30 \mathrm{~ms}$ (Lorimer 2009). The definition is not accurate as the requirement is to separate out a class of RPPs that have gone through different evolutionary pathways involving long-lived binary systems and a 'recycling' accretion episode reducing both the spin-period and the magnetic field (Tauris 2011). Fortunately though, the ranges of $\mathrm{P}_{\mathrm{s}}$ and $\mathrm{B}_{\mathrm{s}}$ of these MSRPs clearly place them in a nearly disjoint region of the $\mathrm{P}_{\mathrm{s}}-\mathrm{B}_{\mathrm{s}}$ plane from the normal radio pulsars (Fig.1).

3. The black widow (BW) and the redback (RB) pulsars actually comprise a special class of binary MSRPs. They are so named because they are in the process of destroying their companions through strong pulsar winds and are likely to be left as isolated millisecond pulsars (Kluzniak et al. 1988; Phinney et al. 1988).

Accretion Powered Pulsars (APP) : Powered by material accretion from a companion, these are classified as High-Mass X-ray Binaries (HMXB) or Low-Mass $\mathrm{X}$-Ray Binaries (LMXB) depending on the mass of the donor star.

1. Neutron stars in HMXBs with $B_{s} \sim 10^{12} \mathrm{G}$ have $O$ or $B$ type companions and typically show up as X-ray pulsars (Caballero \& Wilms 2012). Interaction of the accreting material with the strong magnetic field of the neutron star shows up as broad absorption lines known as Cyclotron Resonance Scattering Features (CRSF) in the hard $X$-ray spectrum of HMXBs. Strength of the magnetic field can be estimated from CRSFs as follows -

$E_{c}=11.6 \mathrm{keV} \times \frac{1}{1+z} \times \frac{B}{10^{12}} \mathrm{G} ;$

where $E_{c}$ is the centroid energy and $z$ is the gravitational red-shift.

2. Neutron stars in LMXBs, on the other hand, have magnetic fields significantly weakened $\left(B<10^{11} \mathrm{G}\right)$ through an extended phase of accretion. Among all the LMXBs harbouring neutron stars - the accreting millisecond X-ray pulsars (AMXP) and the accreting millisecond $\mathrm{X}$-ray bursters (AMXB) are of special interest as they are understood to be immediate precursors of MSRPs and are expected to go into a radio pulsar phase upon the cessation of accretion. The magnetic field in these systems is usually obtained based upon an estimate of the inner truncation radius of the accretion disc, since at that radius the disk pressure is balanced by the pressure of the magnetic field.

Internal Energy Powered Neutron Stars (IENS) : The mechanism of energy generation is not obvious for this class of neutron stars and is suspected to be related to some internal process, like the decay of a strong magnetic field or the residual heat from the progenitor supernova. 


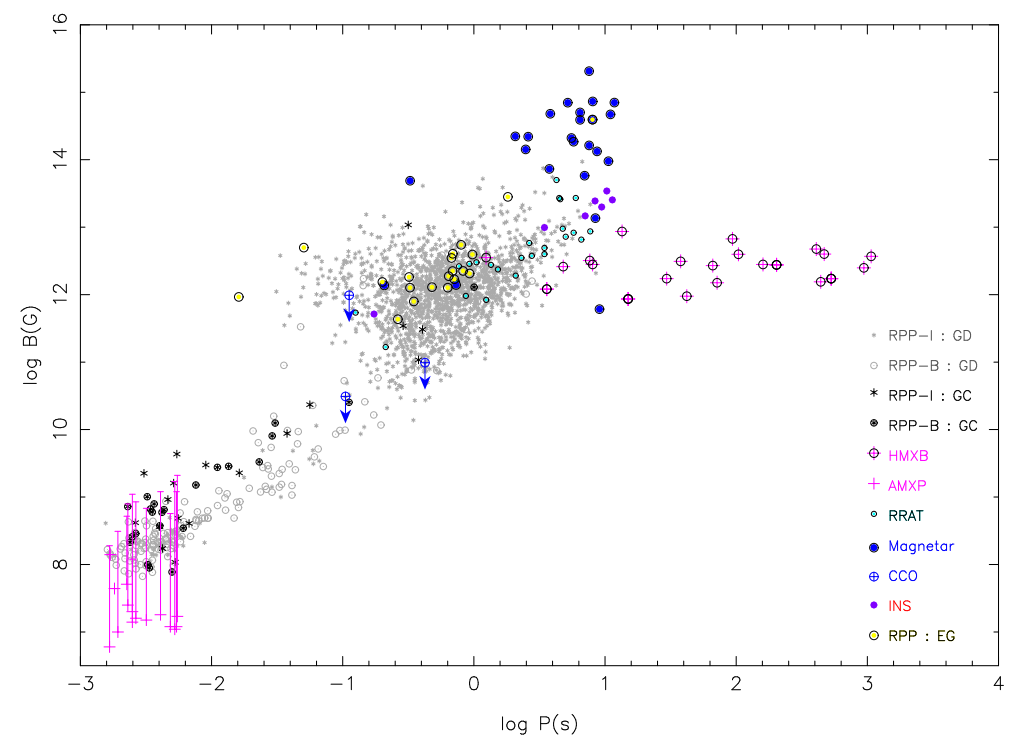

Figure 1: The Menagerie : Different observational classes of neutron stars in the $\mathrm{P}_{\mathrm{s}}-\mathrm{B}_{\mathrm{s}}$ plane.

Legends : I/B - isolated/binary, GC - globular cluster, GD - galactic disc, EG - extra-galactic objects.

Data : RPP - http://www . atnf.csiro.au/research/pulsar/psrcat/,

RRAT - http://astro.phys.wvu.edu/rratalog/,

Magnetar - http://www.physics.mcgill.ca/ pulsar/magnetar/main.html,

AMXP - Patruno \& Watts (2012), Mukherjee et al. (2015); HMXB - Caballero \& Wilms (2012), INS - Haberl

(2007), Kaplan \& van Kerkwijk (2009); CCO - Halpern \& Gotthelf (2010), Ho (2013).

1. Magnetars are thought to be young, isolated neutron stars, that manifest themselves as soft gamma ray repeaters (SGR) and anomalous X-ray pulsars (AXP). It is believed that the main energy source of these objects is the decay of super-strong magnetic fields (magnetar model) (Thompson \& Dunca 1996).

2. X-ray bright central compact objects (CCO) are characterised by an absence both of associated nebulae and exceptionally low magnetic fields (B $\left.10^{10} \mathrm{G}\right)$. Likely, a phase of hypercritical accretion immediately after the birth of the neutron star buries the original field to deeper regions of the crust (Viganò \& Pons 2012).

3. The seven isolated neutron stars (INS), popularly known as the Magnificent Seven, are optically faint and have blackbody-like X-ray spectra $\left(T \sim 10^{6} \mathrm{~K}\right)$. They are probably like ordinary pulsars (with the pulsar beam turned away from us) but a combination of strong magnetic field and spatial proximity make them visible in $\mathrm{X}$-rays.

\section{Magnetic Field : Evolution in Binaries}

As mentioned before the evolution of magnetic field is one of the central ingredients in understanding the interconnections between different observational classes of neutron stars. In this section we discuss some of the well-developed formalisms that have been reasonably successful in explaining the field evolution in neutron stars engaged in active mass accretion, as indicated by observations ${ }^{1}$.

Diamagnetic screening : A number of mechanisms, for accretion-induced field decay, has been suggested over the years amongst which this probably is the least understood process. In an accreting neutron star, material is channelled along the magnetic field lines from the accretion disc forming accretion columns on the magnetic poles (Ghosh \& Lamb 1978; Basko \& Sunyaev 1976). The matter accumulated at the base of such columns can significantly distort the local magnetic field (Melatos \& Phinney 2001; Payne \& Melatos

\footnotetext{
${ }^{1}$ The evolution of magnetic field in neutron stars, in particular the question of MSRP generation in LMXBs, has been one of the primary focus area of the astrophysics group, RRI, under the leadership of G. Srinivasan
} 

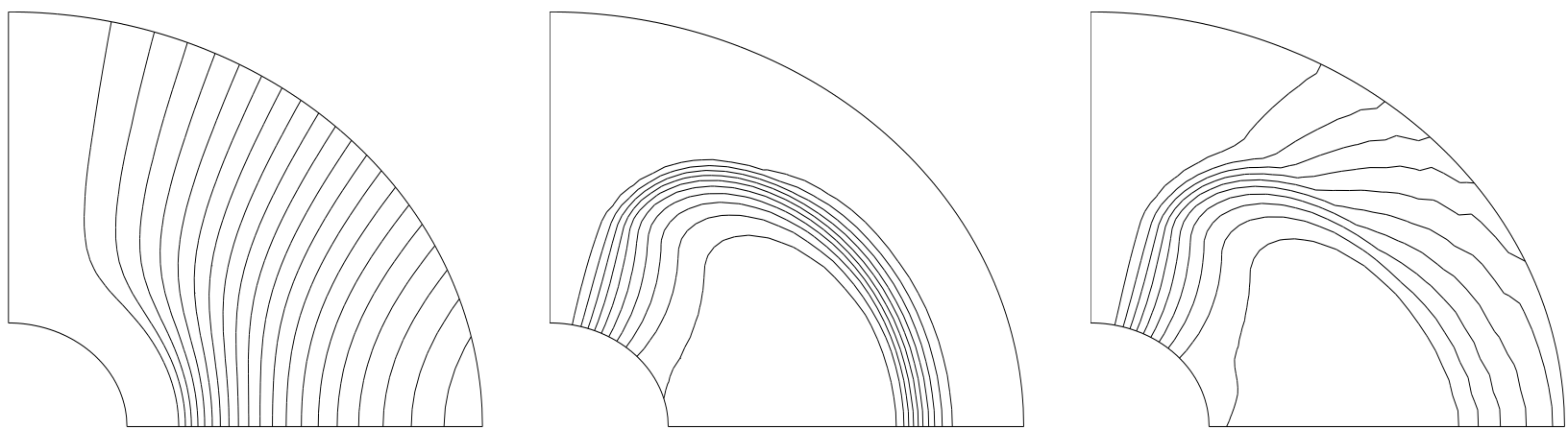

Figure 2: The initial configuration of crustal field in an idealised region defined by $0.25 \leq r / R_{\mathrm{ns}} \leq 1$ ( $r$ - radial coordinate, $R_{\mathrm{ns}}$ - radius of the neutron star) and $0 \leq \theta \leq \pi / 2$. The second and the third panel correspond to the field configuration at a later time without and with magnetic buoyancy. See Choudhuri \& Konar (2002) and Konar \& Choudhuri (2004) for details.

2004; Mukherjee \& Bhattacharya 2012). In particular, the field could get buried under a mountain of accreted plasma (Bisnovatyi-Kogan \& Komberg 1974; Blandford, Decampli, \& Konigl 1979; Woosley \& Wallace 1982; Hameury et al. 1983; Romani 1990; Brown \& Bildsten 1998). When the amount of accreted matter is so large that the material pressure at the bottom of the column exceeds the magnetic pressure, the mountain spreads laterally, transporting the polar magnetic flux towards the equator and finally dissipating them below the surface (Cumming, Zweibel, \& Bildsten 2001; Melatos \& Phinney 2001; Choudhuri \& Konar 2002; Konar \& Choudhuri 2004; Payne \& Melatos 2004; Payne $\&$ Melatos 2007).

When the accreting material flows laterally from the polar caps to lower latitudes, magnetic field lines are dragged by the flow, creating horizontal field at the expense of the vertical ones. Such horizontal magnetic fields are known to be subject to magnetic buoyancy and may cause the field to pop back up to the surface (Spruit 1983) (see Fig.2 illustrating this behaviour). Because of this, there is no clear consensus on whether large scale field burial is possible without re-emergence (Cumming, Zweibel, \& Bildsten 2001) or if MHD instabilities would inhibit deformation of field lines required to reduce the apparent dipole moment (Mukherjee, Bhattacharya, \& Mignone 2013a; Mukherjee, Bhattacharya, \& Mignone 2013b).

\section{Ohmic dissipation :}

As mentioned before, the micro-physics of field evolution depends crucially on the nature of the currents supporting the magnetic field. It is a case of straightforward Ohmic dissipation in an accretion heated crust, if the field is supported by crustal currents. However, if the magnetic field exists in the form of Abrikosov

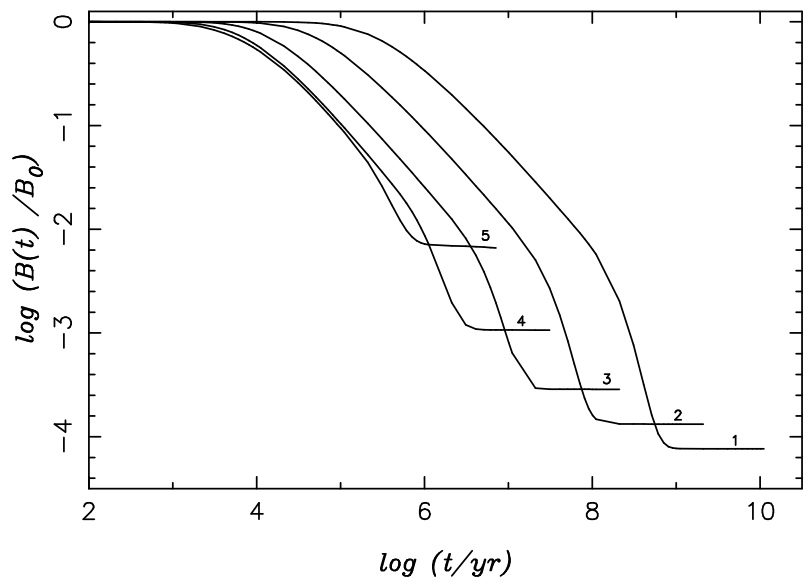

Figure 3: Evolution of the surface magnetic field strength of a neutron star as a result of mass accretion, assuming the crustal currents to be concentrated at a density of $10^{13} \mathrm{~g} \mathrm{~cm}^{-3}$. Curves 1 to 5 correspond to mass accretion rates of $\dot{M}=$ $10^{-12}, 10^{-11}, 10^{-10}, 10^{-9}, 10^{-8} \mathrm{M}_{\odot} / \mathrm{yr}$ respectively. See Konar \& Bhattacharya (1997) for details.

fluxoids in the super-fluid regions of the core, then a prior phase of flux expulsion (to the metallic crust) would be required.

In an accretion-heated crust, the decay takes place principally as a result of rapid dissipation of currents due to a decrease in the electrical conductivity and a resultant reduction in Ohmic dissipation time scale (Geppert \& Urpin 1994; Urpin \& Geppert 1995; Urpin \& Geppert 1996; Konar \& Bhattacharya 1997; Konar \& Bhattacharya 1999a; Cumming, Arras, \& Zweibel 2004). Ohmic decay of current loops for a permanent decrease in the field strength happens according to the induction 


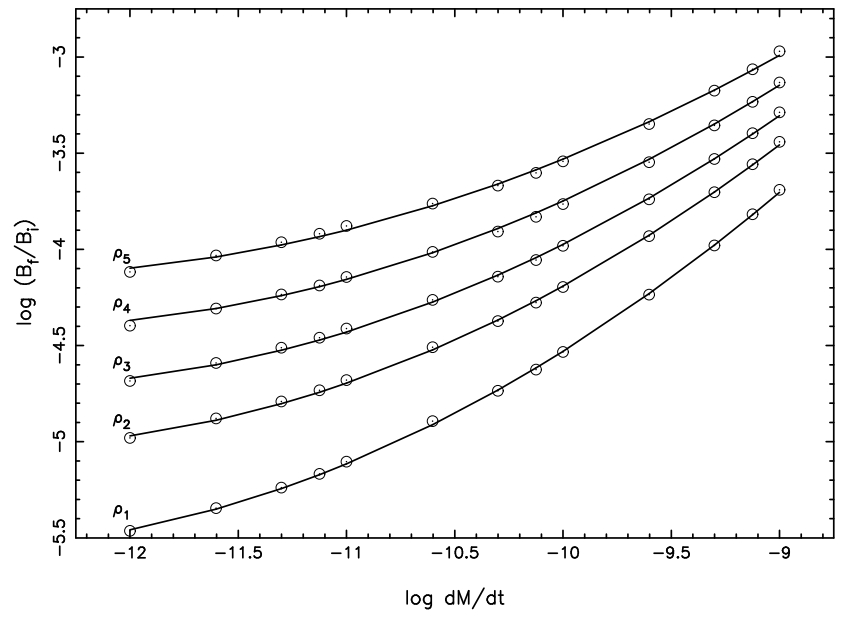

Figure 4: The ratio of the final to initial surface field obtained for given values of accretion rate in $\mathrm{M}_{\odot} / \mathrm{yr}$. Curves marked by $\rho_{1}, \ldots, \rho_{5}$ respectively correspond to densities of $10^{11}, 10^{11.5}, 10^{12}, 10^{12.5}, 10^{13} \mathrm{~g} \mathrm{~cm}^{-3}$, where the currents supporting the magnetic field are concentrated. See Konar \& Bhattacharya (1997) for details.

equation, given by -

$\frac{\partial \mathbf{B}}{\partial t}=\nabla \times(\mathbf{v} \times \mathbf{B})-\frac{\mathbf{c}^{2}}{\mathbf{4} \pi} \nabla \times\left(\frac{1}{\sigma} \nabla \times \mathbf{B}\right)$.

In deeper layers of the crust, field decay is governed essentially by the electrical conductivity $\sigma$, and the radially inward material velocity $\mathbf{v}\left(\propto \dot{M} / \mathbf{r}^{2}\right)$. In turn, $\sigma$ is dependent on $\rho_{c}$, the density at which current carrying layers are concentrated, the impurity content $Q$ and the temperature of the crust $T_{c}$ (again decided by the mass accretion rate $\dot{M}$ ).

Accretion-induced heating reduces $\sigma$ and consequently the Ohmic decay time-scale inducing a faster decay. At the same time material movement, caused by the deposition of matter on top of the crust, pushes the original current carrying layers into deeper and denser regions where higher conductivity slows the decay down. Ultimately the decay stops altogether when the original crust is assimilated into regions with effectively infinite conductivity (Fig.3). Therefore, the final saturation field of an accreting neutron star depends entirely upon the initial magnetic field, the initial $\rho_{c}$ and $\dot{M}$ which determine both $T_{c}$ and $\mathbf{v}$ (Fig.4). Once the surface field freezes into its final value the equilibrium spin-period is obtained by (Alpar et al. 1982; Chen \& Ruderman

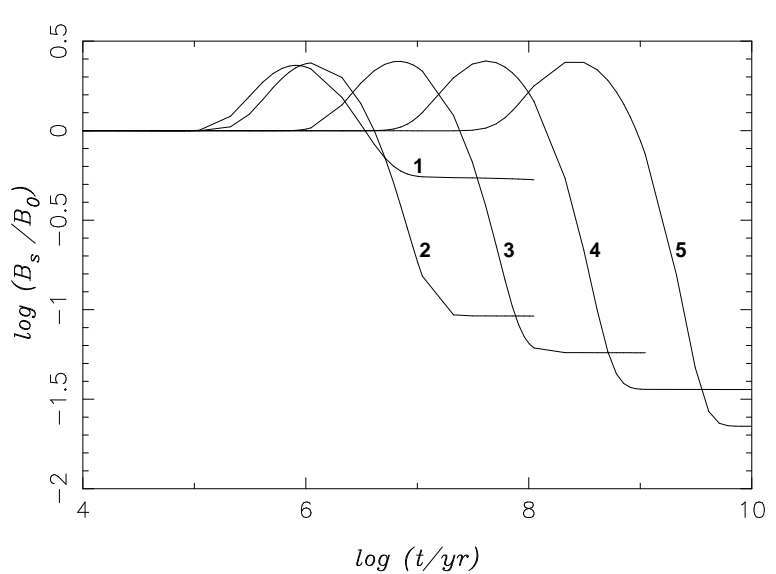

Figure 5: Evolution of the surface magnetic field for an expelled flux. The curves 1 to 5 correspond to $\dot{M}=$ $10^{-9}, 10^{-10}, 10^{-11}, 10^{-12}, 10^{-13} \mathrm{M}_{\odot} \mathrm{yr}^{-1}$. See Konar \& Bhattacharya (1999b) for details.

1993):

$$
\begin{gathered}
P_{\text {eq }} \simeq 1.9 \mathrm{~ms}\left(\frac{B_{\text {final }}}{10^{9} \mathrm{G}}\right)^{6 / 7}\left(\frac{M_{n s}}{1.4 \mathrm{M}_{\odot}}\right)^{-5 / 7} \\
\left(\frac{\dot{M}}{\dot{M}_{\mathrm{Ed}}}\right)^{-3 / 7}\left(\frac{R_{n s}}{10^{6} \mathrm{~cm}}\right)^{16 / 7} .
\end{gathered}
$$

where $\dot{M}_{\mathrm{Ed}}$ is the Eddington accretion rate.

The intrinsic uncertainties associated with the model of Ohmic dissipation are - a) the impurity content of the crust, and b) the exact location of the current carrying layers. In accretion heated crust, the effect of impurities can be entirely neglected and the Ohmic dissipation model can be used to constrain the location of the current carrying layers inside a neutron star. Assuming ordinary neutron stars to be born with magnetic fields in the range of $10^{11.5}-10^{13.5} \mathrm{G}$, we find that in order to generate the observed population of MSRPs, the original current carrying layers need to be concentrated at $\rho_{c} \gtrsim 10^{13} \mathrm{~g} \mathrm{~cm}^{-3}$. Once this is done, the constraint on the impurity content can be found. It appears that an impurity concentration of $\sim 0.01$ is consistent with the fact that the magnetic fields of the isolated radio pulsars do not decay significantly over a time-scale of $10^{6} \mathrm{yrs}$ (Bhattacharya et al. 1992; Hartman et al. 1997).

\section{Spin-down induced flux expulsion :}

Inside the core of a neutron star, the rotation is supported by creation of Onsager-Feynman vortices in the neutron super-fluid whereas the magnetic flux is sustained by Abrikosov fluxoids in the proton superconductor (Baym, Pethick, \& Pines 1969; Ruderman 1972). 


\begin{tabular}{|c|c|c|}
\hline & $\dot{M}\left(\mathrm{M}_{\odot} /\right.$ year $)$ & $\tau$ (year) \\
Wind Accretion & $10^{-14}-10^{-10}$ & $10^{5}-10^{7}$ \\
Roche Contact & $10^{-9}-10^{-8}$ & $10^{3}-10^{5}$ \\
\hline
\end{tabular}

Table 1: Average rates of mass accretion $(\dot{M})$ by the neutron stars and the associated timescales $(\tau)$ during different phases of active mass transfer in typical HMXBs.

There is likely to be a strong inter-pinning between the proton fluxoids and the neutron vortices (Muslimov \& Tsygan 1985a; Sauls 1989). In a spinning down neutron star the neutron vortices migrate outward and by virtue of inter-pinning drag the proton fluxoids along to the outer crust. A binary neutron star interacting with its companion's wind is expected to experience a major spin-down episode in the propeller phase, causing the superconducting core to expel a large fraction of the magnetic flux (Srinivasan et al. 1990). The mechanism of Ohmic decay, being unique to the crustal currents, is invoked for a subsequent dissipation of such flux in the crust (Jahan Miri \& Bhattacharya 1994; Bhattacharya \& Datta 1996; Konar \& Bhattacharya 1999b; Konenkov \& Geppert 2001b; Konenkov \& Geppert 2001a). Due to the fast dissipation of currents in an accretion heated crust, this process may even give rise to a period of initial increase in the surface field (as the core field emerges at the surface) before it starts decreasing (see Fig.5).

\subsection{Neutron Stars in HMXBs}

The theory of the evolution of magnetic field has received quite some attention for neutron stars residing in LMXBs, because of its role in MSRP generation. Unfortunately, similar investigations for HMXB systems are yet to be undertaken. Neutron stars in HMXBs typically have $\mathrm{B}_{\mathrm{s}} \sim 10^{12} \mathrm{G}$ and $\mathrm{O}$ or $\mathrm{B}$ type companions. Since, the timescales in such massive binaries are much smaller than those in LMXBs or IMXBs, the modification of the magnetic field and the spin period would not always be as large as that seen in the LMXBs. Given the average mass accretion rates and timescales of typical HMXBs (see Table. 1) the final values of the surface field and the spin-period, obtained from an Ohmic dissipation model, would typically be mid-range between the ordinary pulsars and the MSRPs (see Table. 2). In particular, even when the surface magnetic field is reduced substantially, there may not be enough time for

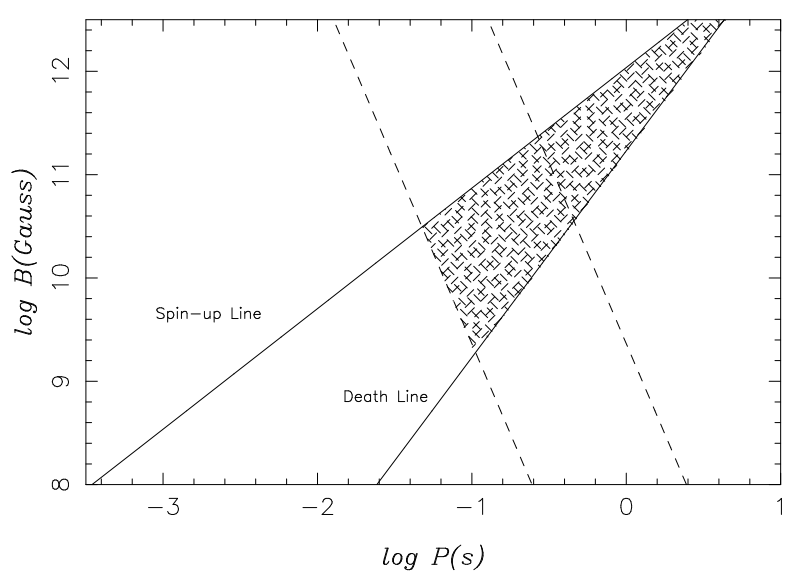

Figure 6: The recycled pulsars from HMXBs are expected to lie within the hatched region of this diagram, assuming the initial surface magnetic field, $\mathrm{B}_{\mathrm{s}}$, to be $\approx 10^{12} \mathrm{G}$. The dashed lines are indicative of maximum achievable spin-up, the upper and lower lines corresponding to accreted masses of $10^{-4}$ and $10^{-3} \mathrm{M}_{\odot}$. For details please see Konar \& Bhattacharya (1999a).

\begin{tabular}{|l|l|}
\hline$\Delta \mathrm{M}\left(\mathrm{M}_{\odot}\right)$ & $10^{-6}-10^{-3}$ \\
$\Delta \mathrm{L}$ (erg.s) & $10^{45}-10^{48}$ \\
$\mathrm{~B}_{\mathrm{i}}(\mathrm{G})$ & $10^{11.5}-10^{13.5}$ \\
$\mathrm{~B}_{\mathrm{f}}(\mathrm{G})$ & $10^{8.5}-10^{13.0}$ \\
$\mathrm{P}_{\mathrm{f}}(\mathrm{s})$ & $\sim 0.1-10.0$ \\
\hline
\end{tabular}

Table 2: The total mass accreted $(\Delta \mathrm{M})$ and the total angular momentum $(\Delta \mathrm{L})$ acquired by a neutron star in an HMXB that can be obtained by assuming the $\dot{M}$ and $\tau$ values given in Table.1. The corresponding final surface field $\left(\mathrm{B}_{\mathrm{f}}\right)$ and spin-period $\left(\mathrm{P}_{\mathrm{f}}\right)$ have been calculated assuming the initial field value $\left(\mathrm{B}_{\mathrm{i}}\right)$ to lie in the typical range appropriate for ordinary non-recycled radio pulsars. 
the pulsar to reach the equilibrium spin-period. Therefore, the HMXBs are likely to produce low-field pulsars with large spin-periods, perhaps close to the death line, as has been shown in Fig.6 (for details see Konar \& Bhattacharya (1999a)). This can also be understood in a different way. A Gaussian decomposition of the spinperiod histogram of all known radio pulsars is shown in Fig.7. The populations of ordinary radio pulsars and the MSRPs clearly stand out. But, it appears that there could be one or more smaller clearly identifiable populations hidden inside. The probability of having just one more population, midway between the ordinary pulsars and the MSRPs, appears to be maximum. The location of this midway population strongly suggests that some of the pulsars 'recycled' in HMXBs would actually be injected into the main island of ordinary pulsars. Interestingly, a similar conclusion was arrived at by Deshpande, Ramachandran, \& Srinivasan (1995a) and Deshpande, Ramachandran, \& Srinivasan (1995b) where they considered the current of pulsars in the $\mathrm{B}_{\mathrm{s}}-\mathrm{P}_{\mathrm{s}}$ plane.

\subsection{Neutron Stars in LMXBs, Millisecond Pulsars}

MSRPs are understood to attain their fast spin and low magnetic field strength through accretion in LMXBs (Alpar et al. 1982; Radhakrishnan \& Srinivasan 1982). These are often found in binaries with evolved, lowmass companions (Bhattacharya \& van den Heuvel 1991) which are thought to be the descendants of LMXBs (Helfand, Ruderman, \& Shaham 1983; Savonije 1983; Paczynski 1983; Joss \& Rappaport 1983). This process of spin-up with attendant accretion-induced field decay of ordinary pulsars to the MSRP class is known as 'recycling'. Therefore, a typical progenitor system of binary MSRPs is expected to be a neutron star accreting from a companion. The discovery of accreting millisecond X-ray pulsars (Wijnands \& van der Klis 1998) and transitional millisecond pulsars (Papitto et al. 2013) has provided confirmation to this evolutionary picture.

The term millisecond pulsar has usually been reserved for 'recycled' pulsars with ultra-fast rotation $\left(\mathrm{P}_{\mathrm{s}}<30\right.$ $\mathrm{ms})$ and a weak magnetic field $\left(\mathrm{B}_{\mathrm{s}} \sim 10^{8}-10^{9} \mathrm{G}\right)$. This definition primarily uses a condition on $\mathrm{P}_{\mathrm{s}}$. Though the most reasonable criterion to define an MSRP should be to use both $\mathrm{P}_{\mathrm{s}}$ and $\dot{P}$ since the evolution of these two parameters are intertwined in the formation process of MSRPs. An effort was made to to use such a relation for the MSRPs in the Galactic disc, assuming them to be recycled through straightforward LMXB evolution (Story, Gonthier, \& Harding 2007). But that is not true of all MSRPs. Moreover, for many MSRPs (in particular for those in globular clusters) $\dot{P}$ determination suf- fers from proper motion contamination and the values may not be entirely reliable even when measurements are available.

On the other hand, there is some justification to use the classical $P \lesssim 20-30 \mathrm{~ms}$ condition to define a MSRP. Fig.7 shows a histogram of the spin-periods of all known galactic radio pulsars. As explained in Sec.3.1, the probability density of the spin-periods is decomposed using a Gaussian mixture model and the data is best fit by a combination of three distinct Gaussian components. The left-most Gaussian is identified with the MSRP population as the overall probability density shows a prominent dip around $P \sim 20-30 \mathrm{~ms}$ clearly separating this population out from the rest.

In recent years, we have seen millisecond pulsars appearing in a variety of types. Like in the case of the overall neutron star population, it is likely that these different types represent either different phases or different pathways of evolution. The millisecond pulsar types that show distinctly different characteristics from each other are - ordinary MSRP, BW \& RB, AMXP, and perhaps the newly emerging class of Gamma-ray pulsars (which may or may not have radio counterparts).

The AMXPs have, of course, been identified as immediate precursors to the MSRPs and are expected to move to the radio emitting phase upon cessation of active material accretion. This conjecture has now been firmly established with the detection of three transient pulsars, PSR J1023+0038, PSR J1227-4853 and PSR J1824-2452I. All of these objects alternate between a non-accreting radio emitting phase and an accreting X-ray phase (Archibald et al. 2009; Roy et al. 2015; Papitto et al. 2013).

The population of AMXPs has been growing rapidly over the last few years, taking the count to 35 (inclusive of standard AMXPs and AMXBs) (Mukherjee et al. 2015; Konar, Mukherjee, \& Bhattacharya 2017). These objects typically belong to ultra-compact binaries, with highly evolved white or brown dwarf companions (Deloye \& Bildsten 2003), undergoing mass transfer at very low rates of accretion from their low-mass companions. The typical values of $\dot{M}$ observed in AMXP/AMXBs is in the range $10^{-10}-10^{-12} \mathrm{M}_{\odot} / \mathrm{yr}$. It is understood that objects like SAX J1808 (the first AMXP to be discovered) are progenitors of fast MSRPs with very short orbital periods (or end up isolated, given the BW nature of SAX J1808) which have undergone very long periods (Gyr) of accretion at very low rates $\left(\dot{M} \sim 10^{-11} \mathrm{M}_{\odot} / \mathrm{yr}\right)$ (Bildsten \& Chakrabarty 2001).

The AMXPs are primarily detected by their coherent or nearly coherent pulsations in persistent X-ray emission. 

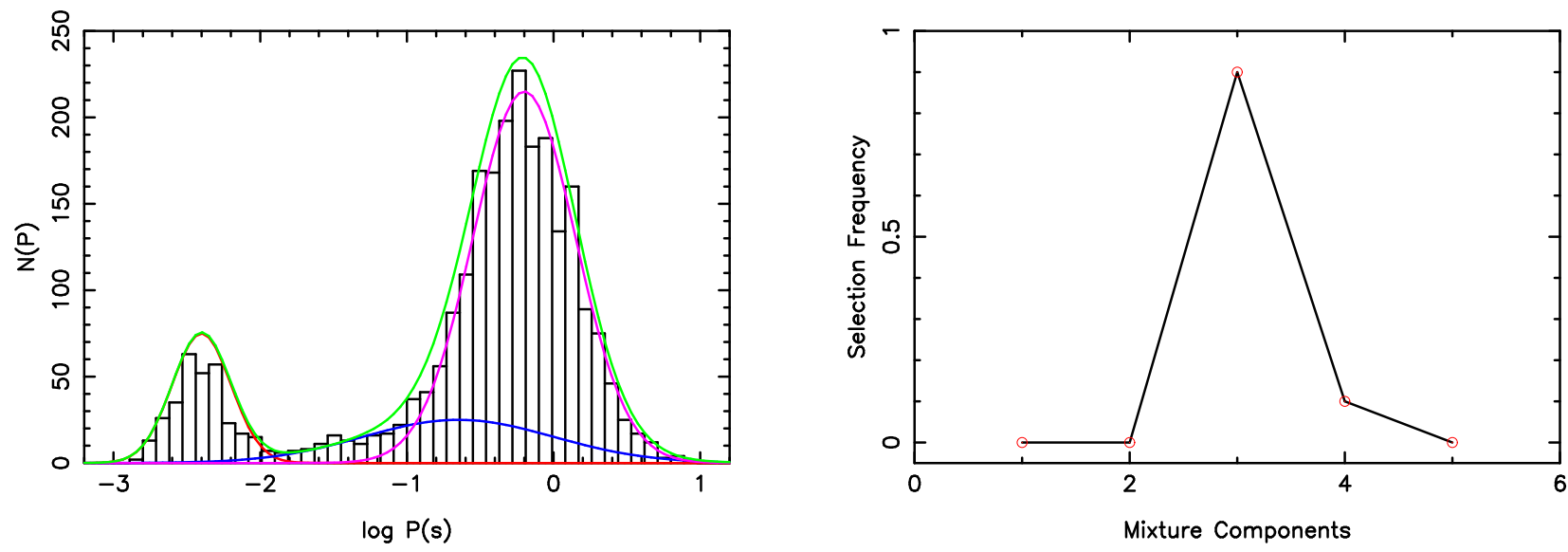

Figure 7: Histogram of the spin-period $\left(\mathrm{P}_{\mathrm{s}}\right)$ of known galactic radio pulsars. A Gaussian decomposition method has been used to find the existence of uniquely different classes in this population. The left-hand panel shows decomposition of the entire population into three Gaussian components. The righthand panel shows the maximum likelihood for a particular decomposition with a given number of components. It is evident that a 3-component decomposition is the likeliest of all. The data is taken from http://www . atnf.csiro.au/research/pulsar/psrcat/.

\begin{tabular}{|l|c|c|c|c|}
\hline & $\begin{array}{c}<\mathrm{P}_{\mathrm{s}}> \\
\mathrm{ms}\end{array}$ & $\begin{array}{c}<\mathrm{B}_{\mathrm{s}}> \\
10^{8} \mathrm{G}\end{array}$ & $<\mathrm{P}_{\mathrm{o}}>$ & $\begin{array}{c}<\mathrm{M}_{\mathrm{c}}> \\
\mathrm{M}_{\odot}\end{array}$ \\
$\begin{array}{l}\text { MSRP } \\
\text { Galactic Disc : }\end{array}$ & 5.99 & 4.64 & $\overline{ }$ & \\
$\begin{array}{l}\text { isolated } \\
\text { binary }\end{array}$ & 7.68 & 8.67 & $37.10 \mathrm{~d}$ & $\overline{0.28}$ \\
$\begin{array}{l}\text { BW + RB } \\
\text { Globular Clusters : }\end{array}$ & 2.90 & 1.80 & $7.09 \mathrm{hr}$ & 0.081 \\
$\begin{array}{l}\text { isolated } \\
\text { binary }\end{array}$ & 5.89 & 13.62 & $\overline{8.67 \mathrm{~d}}$ & $\overline{0.18}$ \\
AMXP + AMXB & 2.77 & $0.27^{*}$ & $4.61 \mathrm{hr}$ & 0.14 \\
\hline
\end{tabular}

Table 3: A summary of some of the average properties of known millisecond pulsars. The data for the MSRP is taken from the ATNF online pulsar catalogue (Manchester et al. 2005). For AMXPs, the magnetic field average has only been calculated as the mean of the estimated lower limits (Mukherjee et al. 2015). 
The main characteristic traits of these pulsars are the following.

- They are components of transient ultra-compact binaries with $\mathrm{P}_{\text {orb }} \sim 40 \mathrm{~m}-20$ hrs.

- They usually have ultra low-mass companions with $\mathrm{M}_{c}<0.1 \mathrm{M}_{\odot}$ (mostly).

- There appears to be an absence of type-I X-ray bursts and $\mathrm{H} \& \mathrm{He}$ lines in outburst of ultra-compact $\left(\mathrm{P}_{\text {orb }}<1 \mathrm{hr}\right.$ ) AMXPs suggesting that they have evolved dwarf companions.

- A number of systems are observed to have orbital periods below the minimum period for systems with hydrogen donor $\left(\mathrm{P}_{\text {orb }}<80 \mathrm{~min}\right)$.

- The inferred long-term $\dot{M}$ and $\mathrm{L}_{\text {outburst }}$ happen to be the lowest amongst all LMXB systems.

On the other hand, the AMXBs exhibit nearly coherent millisecond oscillations during thermonuclear typeI X-ray bursts. As the X-ray burst evolves, the oscillation frequency typically approaches an asymptotic value which is stable for a given source from burst to burst. This asymptotic frequency is thought to trace within a few $\mathrm{Hz}$ the spin frequency of the neutron star (Strohmayer et al. 1996) as has already been observed for the AMXPs - SAX J1808-3658 (Chakrabarty et al. 2003), XTE J1814-338 (Strohmayer et al. 2003), Aql X1 (Casella et al. 2008) etc.

An important point about AMXPs is that a number of them, including the famous SAX J1808 (Bildsten \& Chakrabarty 2001), show clear black widow traits. In fact, each of the three transient pulsars (PSR J1023+0038 PSR J1227-4853, PSR J1824-2452I) is an RB system, with a main-sequence star as a companion. It is understood that these AMXP/transient systems displaying BW/RB behaviour would eventually join the ranks of the isolated, rather than binary, radio millisecond pulsars with total evaporation of their companions. There is some qualitative support for this expectation. Some of the important physical parameters of different millisecond pulsar classes have been summarised in Table.3. It can be seen that the average orbital period of the $\mathrm{BW} / \mathrm{RB}$ radio pulsars is much smaller than that of the binary MSRPs. In fact, it is much closer to the average orbital period of the AMXPs and indicates that these two classes are expected to follow similar evolutionary paths.

However, a comparison of the spin-period distribution of the AMXPs and the isolated MSRPs reveals that they are quite different (see Fig.8), with MSRPs having much

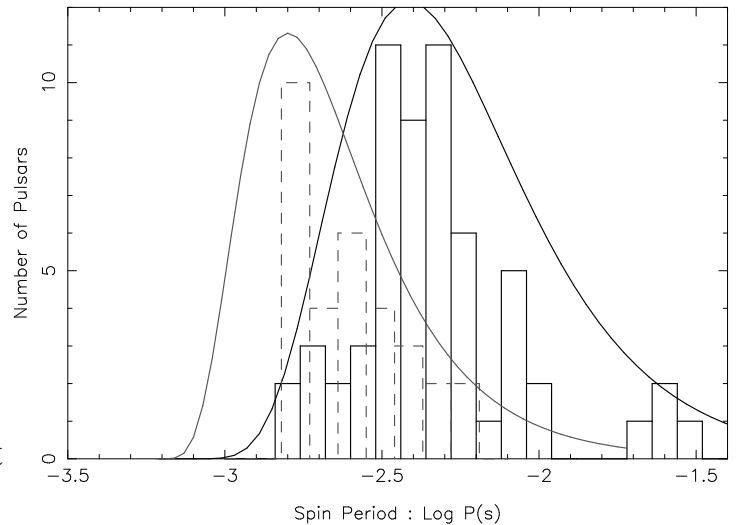

Figure 8: The spin-period histograms and the best-fit curves for AMXPs and the isolated MSRPs.

larger average spin-period (see Table.3). At first glance, this may indicate that the observed AMXPs can not be similar to the progenitors of the isolated MSRPs observed presently. Interestingly, this discrepancy is easily resolved if we assume that the AMXPs should, in fact, be treated like 'zero-age' isolated MSRPs. Most of the AMXP systems are observed to have very low mass-accretion rates and are likely to be just at the end of the accretion phase (as suggested by the intermittent accretion seen in transient pulsars). Therefore, as soon as the accretion stops these AMXPs would become fullfledged 'zero-age' MSRPs. On the other hand, once the active accretion phase stops, the magnetic field should stop evolving and freeze to its final value (Konar 1997). It is then reasonable to assume that the AMXPS (or 'zero-age' isolated MSRPs) to have magnetic field distribution similar to that seen in isolated MSRPs. Evolving such a population for $10^{9}$ years we find that the distribution of the final spin periods closely resemble the spin period distribution of the isolated MSRPs (see Fig.9), confirming that the AMXPs are indeed direct progenitors of the isolated MSRPs.

A large number of pulsars has been detected in the globular clusters in recent years and they appear to have somewhat different characteristics than the pulsars observed in the Galactic disc (see Table.3). To begin with, it is found that the average orbital period of the binary millisecond pulsars in the globular clusters is neither similar to that in the disc nor to the AMXP-BW/RB set. This itself is an indication that the binary evolution in the clusters are probably different. The conditions prevailing in a globular cluster are rather different from those in the Galactic disc primarily due to the extremely high stellar densities in the clusters. One of the obvious effects of this high density is a dramatic increase in the number of binaries, as well as in the rate of close stellar encounters allowing for many different 


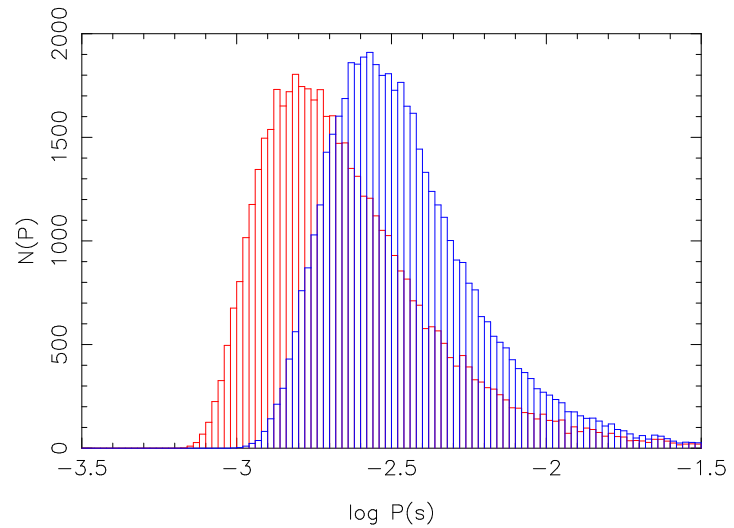

Figure 9: A simulated population of zero-age isolated MSRPs with the spin-distribution of AMXPs (red histogram) is evolved for $10^{9}$ years assuming the magnetic field remains constant. It evolves into a population (blue histogram) that closely resemble the present day isolated MSRP population.

channels for binary formation. As a consequence, most binaries are not primordial in globular clusters. For example, the total observed number of LMXBs in globular clusters exceeds their formation rate in the disc by several orders of magnitude, indicating a dynamical origin (Clark 1975). Stellar interactions in the clusters involving a neutron star have been studied by a number of authors (Krolik, Meiksin, \& Joss 1984; Rasio \& Shapiro 1991; Davies, Benz, \& Hills 1992; Davies \& Hansen 1998; Rasio, Pfahl, \& Rappaport 2000; Bagchi et al. 2009; Bagchi \& Ray 2009). Moreover, many of the cluster binaries have properties similar to those of the black widow pulsars seen in the Galactic disk population (King, Davies, \& Beer 2003; Freire et al. 2005).

It is also seen that a subset of slower MSRPs in the globular clusters (for which field measurements are available) appear to have surface magnetic fields that are 2-5 times larger compared to their disc counterparts; even though the spin-periods of this subset of cluster pulsars are similar to the spin-periods of the MSRPs in the disc. Likely, the difference in the surface magnetic field could be due to one or several of the possibilities listed below (Konar 2010):

- There are systematic biases in the $\dot{P}$ (hence $\mathrm{B}_{\mathrm{s}}$ ) measurement for cluster MSRPs.

- The cluster MSRPs are younger and may evolve to a distribution similar to the disc MSRPs with time.

- Preferential recycling of MSRPs in tighter binaries with high rates of attendant mass transfer may actually result in cluster pulsars retaining higher magnetic fields.

\section{Magnetic Field : Isolated Evolution}

The basic physics, underlying the model of magnetothermal evolution developed for the evolution of magnetic field in isolated neutron stars, is essentially the same as in the case of accreting systems. However, in absence of any external factors (like material accretion) the evolution feeds upon itself. On the one hand, the field evolution is sensitively dependent on the microphysics (through transport properties) of the interior. Since the transport properties are temperature-dependent, thermal evolution affects field evolution. On the other hand, the Ohmic dissipation of the field generates heat, modifying thermal evolution, changing transport properties and ultimately affecting the field evolution in a cyclical fashion.

In recent years, there have been observational indications for existence of evolutionary pathways linking different classes of isolated neutron stars (Kaspi 2010). There is a clear overlap between the high magnetic field $\left(\mathrm{B}>4 \times 10^{13} \mathrm{G}\right)$ radio pulsars and the magnetars in the B-P diagram (Fig.[1]). The magnetar-like X-ray burst exhibited by PSR J1846-0258 $\left(B=4 \times 10^{13} \mathrm{G}\right)$ has reinforced the suggestion that such high field radio pulsars are quiescent magnetars. Conversely, it has been suggested that hyper-critical fallback accretion may bury the field to deeper crustal layers thereby reducing the surface field, as seen in the CCOs. Subsequent emergence of this buried field could transform a CCO to an ordinary radio pulsar or even to a magnetar. Therefore different combinations of initial spin-period, magnetic field and submersion depth of the field may very well decide whether a neutron star manifests itself as an ordinary radio pulsar, a magnetar or a $\mathrm{CCO}$ (Viganò \& Pons 2012). Similarly, INSs are observed only in Xray, despite being isolated objects. It is possible that they are actually similar to the RPPs and are not seen as radio pulsars simply due to the misalignment of emission cones with our lines of sight. The neutron stars with strong magnetic fields are expected to remain at a relatively higher temperature due to field decay. This could then explain the high (compared to ordinary radio pulsars) X-ray luminosity of the INSs. Finally, it has been argued that the anomalous braking index of PSR J1734-3333 signifies an increase in its dipolar surface magnetic field and is likely driven by the emergence (perhaps glitch-induced) of a stronger field buried underneath the surface, with timescales depending on submersion depth (Espinoza et al. 2011). If cor- 
rect, this process may chart a pathway for the transition from ordinary radio pulsars to magnetars. It appears that different flavors of the isolated neutron stars could, in fact, be intricately connected through various evolutionary pathways.

One of the important ingredients of the theory of magnetothermal evolution, effective in the early phases of a neutron star's life, is the structure of the magnetic field. Many of the models consider the interaction of the observable poloidal field with a toroidal component buried in the deeper layers of the crust. The magnetic field estimated from the spin-down rate of radio pulsars measures only the large scale dipolar field. However, strong multipole components of the magnetic field have long been thought to play an important role in the radio emission from pulsars (Ruderman \& Sutherland 1975; Krolik 1991; Arons 1998; Deshpande \& Rankin 1998; Rankin \& Deshpande 1998; Gil, Melikidze, \& Mitra 2002a; Gil, Melikidze, \& Mitra 2002b; Asseo \& Khechinashvili 2002; Zane \& Turolla 2005; Gil et al. 2008). In absence of material movement (as can be expected in isolated neutron stars) each multipole component evolves separately for purely poloidal field structures. As higher multipole components dissipate much faster than the dipole, it is expected that over long time-scales only the dipole field would survive (Mitra, Konar, \& Bhattacharya 1999). Since existence of higher multipoles is absolutely necessary for pulsar activity, it is imperative that the internal magnetic field of a neutron star must have a toroidal component. Of course, existence of toroidal components is also necessary for the stabilisation of poloidal fields in a newly born fluid neutron star before anchoring in the solid crust can work towards stabilising the large scale field.

\section{Discussions}

The evolutionary pathways, linking different observational classes of neutron stars, has been summarised in Fig.10. It is evident that on the fiftieth year of the discovery of the first radio pulsar, we have unearthed more classes than we have been able to link. Much of the pathways, in particular between the isolated variety, is quite uncertain and is still being explored. It is expected that the number of new neutron stars discovered will increase by a large factor in the near future with the advent of multi-messenger astronomy and future telescopes with better sensitivity and wider frequency coverage. Such increase will definitely improve our understanding of some of today's 'uncertain' pathways but almost certainly will throw up newer challenges.

\section{Acknowledgements}

The author has been privileged to begin her academic career in the astrophysics group of Raman Research Institute, Bangalore; then headed by G. Srinivasan and to learn many of the intricacies of neutron star physics from him. Most of the work reported here has been done in collaboration with Dipankar Bhattacharya, Arnab Rai Choudhuri, Dipanjan Mitra and Dipanjan Mukher$\mathrm{jee}^{2}$. The author is supported by a grant (SR/WOSA/PM-1038/2014) from DST, Government of India.

\section{References}

Alpar M. A., Cheng A. F., Ruderman M. A., Shaham J., 1982, Nature, 300, 728

Archibald A. M. et al., 2009, Science, 324, 1411

Arons J., 1998, in Shibazaki N., ed, Neutron Stars and Pulsars:

Thirty Years after the Discovery, p. 339

Asseo E., Khechinashvili D., 2002, MNRAS, 334, 743

Backer D. C., Kulkarni S. R., Heiles C., Davis M. M., Goss W. M., 1982, Nature, 300, 615

Bagchi M., Dey J., Konar S., Bhattacharya G., Dey M., 2009, New A, 14, 37

Bagchi M., Ray A., 2009, ApJ, 693, L91

Basko M. M., Sunyaev R. A., 1976, MNRAS, 175, 395

Baym G., Pethick C., Pines D., 1969, Nature, 224, 673

Bhattacharya D., 2002, Journal of Astrophysics and Astronomy, 23, 67

Bhattacharya D., Datta B., 1996, MNRAS, 282, 1059

Bhattacharya D., Srinivasan G., 1995, X-ray Binaries, 0, 495

Bhattacharya D., van den Heuvel E. P. J., 1991, Phys. Rep., 203, 1

Bhattacharya D., Wijers R. A. M. J., Hartman J. W., Verbunt F., 1992, A\&A, 254, 198

Bildsten L., Chakrabarty D., 2001, ApJ, 557, 292

Bisnovatyi-Kogan G. S., Komberg B. V., 1974, Soviet Ast., 18,217

Blandford R. D., Decampli W. M., Konigl A., 1979, in Bulletin of the American Astronomical Society, Vol. 11, Bulletin of the American Astronomical Society, p. 703

Brown E. F., Bildsten L., 1998, ApJ, 496, 915

Caballero I., Wilms J., 2012, Mem. Soc. Astron. Italiana, 83,230

Casella P., Altamirano D., Patruno A., Wijnands R., van der Klis M., 2008, ApJ, 674, L41

\footnotetext{
${ }^{2}$ All of these people have been associated with the 'Srini era' of RRI astrophysics, in one capacity or another. Except for Dipanjan Mukherjee who worked with Dipankar Bhattacharya, himself a student of Srini, for his PhD thesis.
} 
Accretion Powered Rotation Powered Internal Energy Powered

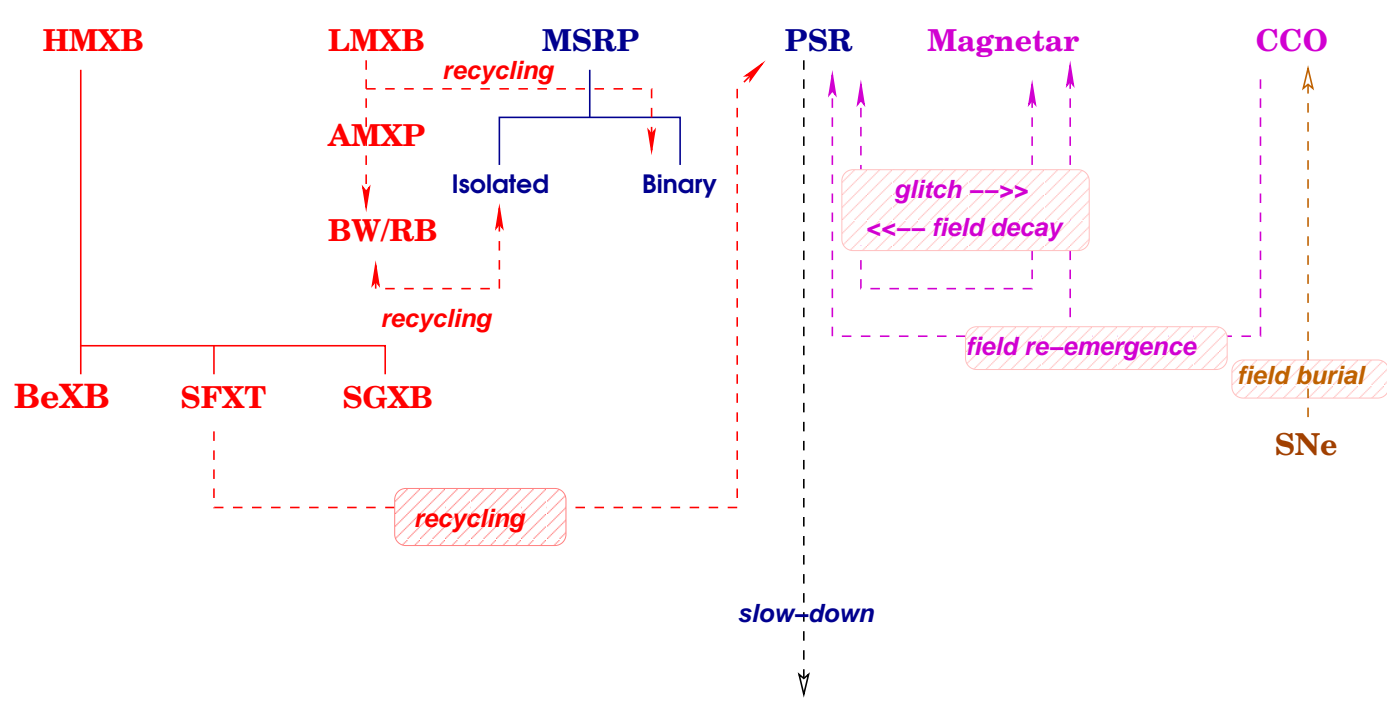

Graveyard

Figure 10: A summary of the evolutionary pathways connecting different observational classes of neutron stars. Wherever put inside a shaded box, the pathway is not a confirmed process yet. (Legends : HMXB - high mass X-ray binary, BeXB - Be X-ray binary, SFXT - super-giant fast X-ray transient, SGXB - super-giant X-ray binary, LMXB - low mass X-ray binary, MSRP - millisecond radio pulsar, AMXP - accreting millisecond X-ray pulsar, BW/RB - black-widow/redback pulsar, PSR - radio pulsar, CCO - central compact object, $\mathrm{SNe}$ - supernova explosion)

Chakrabarty D., Morgan E. H., Muno M. P., Galloway D. K., Wijnands R., van der Klis M., Markwardt C. B., 2003, Nature, 424, 42

Chen K., Ruderman M., 1993, ApJ, 402, 264

Choudhuri A. R., Konar S., 2002, MNRAS, 332, 933

Clark G. W., 1975, ApJ, 199, L143

Cumming A., Arras P., Zweibel E., 2004, ApJ, 609, 999

Cumming A., Zweibel E., Bildsten L., 2001, ApJ, 557, 958

Davies M. B., Benz W., Hills J. G., 1992, ApJ, 401, 246

Davies M. B., Hansen B. M. S., 1998, MNRAS, 301, 15

Deloye C. J., Bildsten L., 2003, ApJ, 598, 1217

Deshpande A. A., Ramachandran R., Srinivasan G., 1995a,

Journal of Astrophysics and Astronomy, 16, 53

Deshpande A. A., Ramachandran R., Srinivasan G., 1995b,

Journal of Astrophysics and Astronomy, 16, 69

Deshpande A. A., Rankin J. M., 1998, in Bulletin of the

American Astronomical Society, Vol. 30, American Astronomical Society Meeting Abstracts, p. 1387

Elfritz J. G., Pons J. A., Rea N., Glampedakis K., Viganò D., 2016, MNRAS, 456, 4461

Espinoza C. M., Lyne A. G., Kramer M., Manchester R. N., Kaspi V. M., 2011, ApJ, 741, L13

Freire P. C. C., Hessels J. W. T., Nice D. J., Ransom S. M., Lorimer D. R., Stairs I. H., 2005, ApJ, 621, 959

Geppert U., Rheinhardt M., 2002, A\&A, 392, 1015

Geppert U., Urpin V., 1994, MNRAS, 271, 490
Ghosh P., Lamb F. K., 1978, ApJ, 223, L83

Gil J., Haberl F., Melikidze G., Geppert U., Zhang B., Melikidze G., Jr., 2008, ApJ, 686, 497

Gil J. A., Melikidze G. I., Mitra D., 2002a, A\&A, 388, 235

Gil J. A., Melikidze G. I., Mitra D., 2002b, A\&A, 388, 246

Glampedakis K., Andersson N., Samuelsson L., 2011, MNRAS, 410, 805

Goldreich P., Reisenegger A., 1992, ApJ, 395, 250

Graber V., Andersson N., Glampedakis K., Lander S. K., 2015, MNRAS, 453, 671

Haberl F., 2007, Ap\&SS, 308, 181

Halpern J. P., Gotthelf E. V., 2010, ApJ, 709, 436

Hameury J. M., Bonazzola S., Heyvaerts J., Lasota J. P., 1983, A\&A, 128, 369

Hartman J. W., Bhattacharya D., Wijers R., Verbunt F., 1997, A\&A, 322, 477

Helfand D. J., Ruderman M. A., Shaham J., 1983, Nature, 304, 423

Hewish A., Bell S. J., Pilkington J. D. H., Scott P. F., Collins R. A., 1968, Nature, 217, 709

Ho W. C. G., 2013, in IAU Symposium, Vol. 291, IAU Symposium, p. 101

Hollerbach R., Rüdiger G., 2002, MNRAS, 337, 216

Jahan Miri M., Bhattacharya D., 1994, MNRAS, 269, 455

Joss P. C., Rappaport S. A., 1983, Nature, 304, 419

Kaplan D. L., van Kerkwijk M. H., 2009, ApJ, 692, L62 
Kaspi V. M., 2010, Proceedings of the National Academy of Science, 107, 7147

King A. R., Davies M. B., Beer M. E., 2003, MNRAS, 345, 678

Kluzniak W., Ruderman M., Shaham J., Tavani M., 1988, Nature, 334, 225

Konar S., 1997, Evolution of the Magnetic Field in Accreting Neutron Stars

Konar S., 2010, MNRAS, 409, 259

Konar S., 2013, in Astronomical Society of India Conference Series, Vol. 8, Das S., Nandi A., Chattopadhyay I., ed, Astronomical Society of India Conference Series, p. 89

Konar S. et al., 2016, Journal of Astrophysics and Astronomy, 37, 36

Konar S., Bhattacharya D., 1997, MNRAS, 284, 311

Konar S., Bhattacharya D., 1999a, MNRAS, 303, 588

Konar S., Bhattacharya D., 1999b, MNRAS, 308, 795

Konar S., Choudhuri A. R., 2004, MNRAS, 348, 661

Konar S., Mukherjee D., Bhattacharya D., 2017, in prep., 0

Konenkov D., Geppert U., 2000, MNRAS, 313, 66

Konenkov D., Geppert U., 2001a, A\&A, 372, 583

Konenkov D. Y., Geppert U., 2001b, Astronomy Letters, 27, 163

Krolik J. H., 1991, ApJ, 373, L69

Krolik J. H., Meiksin A., Joss P. C., 1984, ApJ, 282, 466

Lorimer D. R., 2009, in Astrophysics and Space Science Library, Vol. 357, Becker W., ed, Astrophysics and Space Science Library, p. 1

Manchester R. N., Hobbs G. B., Teoh A., Hobbs M., 2005, AJ, 129, 1993

Manchester R. N., Taylor J. H., 1977, Pulsars. W. H. Freeman, San Francisco, San Francisco, p. 36

Melatos A., Phinney E. S., 2001, PASA, 18, 421

Mitra D., Konar S., Bhattacharya D., 1999, MNRAS, 307, 459

Mukherjee D., Bhattacharya D., 2012, MNRAS, 420, 720

Mukherjee D., Bhattacharya D., Mignone A., 2013a, MNRAS, 430, 1976

Mukherjee D., Bhattacharya D., Mignone A., 2013b, MNRAS, 435, 718

Mukherjee D., Bult P., van der Klis M., Bhattacharya D., 2015, MNRAS, 452, 3994

Muslimov A. G., Tsygan A. I., 1985a, Soviet Astronomy Letters, 11, 80

Muslimov A. G., Tsygan A. I., 1985b, Ap\&SS, 115, 43

Paczynski B., 1983, Nature, 304, 421

Papitto A. et al., 2013, Nature, 501, 517

Passamonti A., Akgün T., Pons J. A., Miralles J. A., 2017, MNRAS, 465, 3416

Patruno A., Watts A. L., 2012, ArXiv e-prints, 0

Payne D. J. B., Melatos A., 2004, MNRAS, 351, 569

Payne D. J. B., Melatos A., 2007, MNRAS, 376, 609

Phinney E. S., Evans C. R., Blandford R. D., Kulkarni S. R., 1988, Nature, 333, 832

Pons J. A., Miralles J. A., Geppert U., 2009, A\&A, 496, 207
Radhakrishnan V., Srinivasan G., 1982, Current Science, 51, 1096

Rankin J. M., Deshpande A. A., 1998, in Bulletin of the American Astronomical Society, Vol. 30, American Astronomical Society Meeting Abstracts, p. 1309

Rasio F. A., Pfahl E. D., Rappaport S., 2000, ApJ, 532, L47

Rasio F. A., Shapiro S. L., 1991, ApJ, 377, 559

Reisenegger A., 2007, Astronomische Nachrichten, 328, 1173

Reisenegger A., Benguria R., Prieto J. P., Araya P. A., Lai D., 2007, A\&A, 472, 233

Rheinhardt M., Geppert U., 2002, Physical Review Letters, 88, 101103

Romani R. W., 1990, Nature, 347, 741

Roy J. et al., 2015, ApJ, 800, L12

Ruderman M., 1972, ARA\&A, 10, 427

Ruderman M. A., Sutherland P. G., 1975, ApJ, 196, 51

Sang Y., Chanmugam G., 1987, ApJ, 323, L61

Sauls J., 1989, in Ögelman H., van den Heuvel E. P. J., ed, Timing Neutron Stars, p. 457

Savonije G. J., 1983, Nature, 304, 422

Spruit H. C., 1983, in IAU Symposium, Vol. 102, Stenflo J. O., ed, Solar and Stellar Magnetic Fields: Origins and Coronal Effects, p. 41

Srinivasan G., Bhattacharya D., Muslimov A. G., Tsygan A. J., 1990, Current Science, 59, 31

Story S. A., Gonthier P. L., Harding A. K., 2007, ApJ, 671, 713

Strohmayer T. E., Markwardt C. B., Swank J. H., in't Zand J., 2003, ApJ, 596, L67

Strohmayer T. E., Zhang W., Swank J. H., Smale A., Titarchuk L., Day C., Lee U., 1996, ApJ, 469, L9

Tauris T. M., 2011, in Astronomical Society of the Pacific Conference Series, Vol. 447, Schmidtobreick L., Schreiber M. R., Tappert C., ed, Evolution of Compact Binaries, p. 285

Thompson C., Duncan R. C., 1996, ApJ, 473, 322

Urpin V., Geppert U., 1995, MNRAS, 275, 1117

Urpin V., Geppert U., 1996, MNRAS, 278, 471

Urpin V., Konenkov D., 2008, A\&A, 483, 223

Urpin V. A., Muslimov A. G., 1992, MNRAS, 256, 261

Urpin V. A., van Riper K. A., 1993, ApJ, 411, L87

Viganò D., 2013, Magnetic fields in neutron stars

Viganò D., Pons J. A., 2012, MNRAS, 425, 2487

Wijnands R., van der Klis M., 1998, Nature, 394, 344

Woosley S. E., Wallace R. K., 1982, ApJ, 258, 716

Zane S., Turolla R., 2005, Advances in Space Research, 35, 1162 